\title{
Vigilancia de la presencia de triatominos mediante gallineros en el sur de Jalisco, México
}

\author{
José Alejandro Martínez-lbarra', Jorge Alejandro Martínez-Grant¹, Miguel Roberto Verdugo-Cervantes', \\ Rafael Bustos-Saldaña', Benjamín Nogueda-Torres² \\ 1 Área de Entomología Médica, Centro Universitario del Sur, Universidad de Guadalajara, Ciudad Guzmán, \\ Jalisco, México \\ 2 Escuela Nacional de Ciencias Biológicas, Instituto Politécnico Nacional, México, D. F., México
}

Introducción. En México, la enfermedad de Chagas es un problema de salud pública. Se han registrado, al menos, 30 especies de triatominos vectores, la mayoría con hábitos domésticos y tendencia a invadir las viviendas humanas.

Objetivo. Evaluar la utilidad de gallineros en la vigilancia de la presencia de triatominos peridomésticos y conocer su situación en el área de estudio.

Materiales y métodos. Se realizó un estudio de campo, experimental, longitudinal, por un año. Se instalaron 50 gallineros en 50 viviendas de tres localidades del municipio de Teocuitatlán de Corona, en el estado de Jalisco, México. Se inspeccionaron los gallineros y las viviendas para saber si aquéllos podían detectar la presencia de triatominos, para estimar la situación vectorial en el área.

Resultados. Se recolectaron significativamente $(p<0,05)$ más especímenes de Meccus longipennis (93\%) que de Triatoma barberi (7\%). Aproximadamente, un tercio $(31,3 \%)$ de los especímenes de $M$. longipennis y ninguno de $T$. barberi se recolectó en los gallineros. $M$. longipennis tuvo un porcentaje de infección por Trypanosoma cruzi de 25,7\%, y $T$. barberi, de 53,2\%. Los meses de primavera y verano fueron en los que se recolectaron más ejemplares de triatominos.

Conclusiones. Los gallineros se consideran una herramienta útil para detectar la presencia de ejemplares de $M$. longipennis, aunque no funcionaron para $T$. barberi. Se confirma la importancia de $M$. longipennis y se establece el posible rol primario de T. barberi como vector en el área de estudio.

Palabras clave: enfermedad de Chagas, Triatominae, vectores de enfermedades, vigilancia, aves de corral, México.

Monitoring triatomid bug (Hemiptera: Reduviidae) presence by sentinel chicken coops in Southern Jalisco State, México

Introduction. Chagas disease is an important public health problem in Mexico. At least 30 Triatominae species, vectors of that disease have been recorded, most with domiciliary habits and tendency to invade human dwellings.

Objective. The usefulness of using chicken coops was evaluated for monitoring peridomestic triatomine presence, identity and infection with Trypanosoma cruzi.

Materials and methods. A year-long, longitudinal field study was designed. Fifty chicken coops were placed in 50 human dwellings on three localities in Teocuitatlán de Corona County, Jalisco State, Mexico. The coops and human dwellings were routinely inspected to determine if the coops alone can monitor for the presence of triatomines.

Results. Significantly $(p<0.05)$ more Meccus longipennis specimens $(93.0 \%)$ than of Triatoma barberi $(7.0 \%)$ were collected. Almost a third (31.3\%) of $M$. Iongipennis and none of $T$. barberi specimens were collected in the coops. Meccus longipennis was found with infections of Trypanosoma cruzi of $25.7 \%$, and $T$. barberi of $53.2 \%$. Most triatomines were collected in spring and summer months.

Conclusions. Chicken coops proved useful tools to detect presence of $M$. longipennis; however, they were not effective for $T$. barberi. The importance of $M$. Iongipennis as a potential vector was confirmed, and the possible primary vector role of $T$. barberi was established as well.

Key words: Chagas disease, triatominae, disease vectors, monitoring, poultry, México 
En México, la enfermedad de Chagas es una de los principales padecimientos transmitidos por vectores, ya que se considera que existen dos millones de casos y 71.000 personas resultan infectadas con Trypanosoma cruzi (Chagas, 1909) por año (1).

Existen, al menos, 30 especies descritas de triatominos en el país, de las cuales, nueve son consideradas como importantes por su contribución al mantenimiento de la enfermedad en humanos y animales (2). La zona occidental del país se considera zona endémica, lo que se ha demostrado por el número de casos presuntivos de enfermedad, detectados serológicamente o mediante las manifestaciones clínicas y por los reportes de los bancos de sangre (3-5).

En diversas áreas de los cuatro estados de la zona occidental se han encontrado siete de las nueve especies de triatominos consideradas como los vectores más importantes de la enfermedad de Chagas en México (2). Entre ellas, Meccus longipennis [(Usinger), 1939] se destaca por su distribución, grado de domiciliación y positividad a T. cruzi (6-10), por lo que se considera la especie vector principal de $T$. cruzi en el estado de Jalisco $(5,7)$.

Por otra parte, si bien Triatoma barberi (Usinger, 1939) se considera un vector secundario en el área $(7,10-12)$, sus altos porcentajes de infección por T. cruzi $(13,14)$ lo hacen un vector merecedor de vigilancia.

Ambas especies se han encontrado muy asociadas con gallinas y pollos $(6-8,11)$, asociación aparentemente benéfica, corroborada por los parámetros biológicos favorables para $M$. longipennis, obtenidos al ser alimentada esta especie en gallina, en condiciones de laboratorio $(15,16)$.

Tal como se ha documentado $(7,17)$, parece que los gallineros fungen como puerta de entrada para la colonización de los domicilios por los triatominos mexicanos. Estas asociaciones de especies de triatominos con aves son similares a las reportadas en Suramérica para especies de esa región, donde

\footnotetext{
Correspondencia:

José Alejandro Martínez-Ibarra, Centro Universitario del Sur, Universidad de Guadalajara, Prolongación Colón S/N, 49000 Ciudad Guzmán, Jalisco, México.

Telefax: (52341) 575 2222, extensión 6079

aibarra@cusur.udg.mx

Recibido: 14/02/09; aceptado:11/08/09
}

los gallineros han tenido un papel preponderante en la domiciliación de las poblaciones de triatominos (18-22). Si bien se ha probado exitosamente el uso de trampas cebadas con ratas y cubiertas con tela adhesiva para la vigilancia de triatominos en Jalisco (7), la dificultad en conseguir el cebo y la tela hacían necesaria la búsqueda de otro método más sencillo para los moradores del área.

Con la intención de proveer a los habitantes de las localidades rurales con una herramienta de fácil hechura y revisión, buscando que ellos mantengan una vigilancia constante de los triatominos y dado el grado de asociación de $M$. longipennis y $T$. barberi con gallinas, se analizó la eficacia de los gallineros para conocer la presencia de estas especies de vectores en el peridomicilio de las viviendas.

\section{Materiales y métodos}

\section{Área de estudio}

El estudio se realizó en el municipio de Teocuitatlán de Corona, Jalisco, $\left(20^{\circ} 06^{\prime} \mathrm{N}, 103^{\circ} 21^{\prime} \mathrm{O}\right)$. Dicho municipio se seleccionó porque ocupa el tercer lugar estatal en casos presuntivos de enfermedad de Chagas y por estar dentro del área de distribución de $M$. longipennis y $T$. barberi; de igual manera, por haberse detectado altos indicadores de infección natural (superiores a 30\%), de infestación (superiores a 65\%) y de riesgo de transmisión de T. cruzi a los humanos (7).

El cálculo del tamaño de muestra así como el análisis de los resultados mediante pruebas de ji al cuadrado se hicieron con el programa Epilnfo 6.04 (23).

Para el estudio se seleccionaron al azar 50 viviendas, ubicadas proporcionalmente en tres pequeñas comunidades (aproximadamente, de 50 casas cada una), distantes $5 \mathrm{~km}$ unas de otras, muy similares entre sí y con indicadores entomológicos similares a los globales del municipio: Agua Honda, Rancho Nuevo y Tehuantepec.

Con el fin de eliminar los ejemplares de triatominos presentes en cada vivienda, y así corroborar la existencia de un fenómeno de invasión activa por triatominos, previamente al inicio del establecimiento de los gallineros, en cada casa se revisó durante una hora/hombre en búsqueda de triatominos, siguiendo el método de Pinchin (24), con el auxilio de un ligero rociamiento (menos de un minuto) con piretroides. Los ejemplares capturados fueron recolectados en recipientes, separados por vivienda y trasladados al laboratorio. 
Posteriormente, en el patio de cada vivienda se instaló un gallinero prefabricado, de $2 \mathrm{~m} \mathrm{x}$ $1 \mathrm{~m} \times 2 \mathrm{~m}$ (longitud, anchura, altura), hecho de alambre recubierto, galvanizado, y resistente a la intemperie. Se ubicaron cerca de las viviendas, preferentemente por fuera de las recámaras, aproximadamente, a un metro de las paredes de éstas. La ubicación de dichos gallineros se realizó de tal forma que la vivienda no fuera obstáculo entre los refugios más comunes (bardas de piedra) (7) de los triatominos y los gallineros.

En cada gallinero se introdujeron 10 gallinas. Dichos gallineros reunían las condiciones internas típicas de los gallineros usados en el área: presencia de cestos tejidos o papeles, trozos de tela o tejidos, así como ramas y palos para el sostenimiento de las aves adentro. Esto se hizo con la finalidad de que los triatominos que se acercaran a alimentarse de las gallinas tuvieran un refugio, para evitar que las gallinas los depredaran, así como para poder conocer de su presencia en el domicilio.

La búsqueda de triatominos en cada gallinero se hizo durante media hora/hombre, cada 15 días (un día en cada ocasión, a mediados y finales de mes), a lo largo de un año. Las viviendas se revisaron con la misma frecuencia, pero por un lapso de una hora/hombre (24). Como medida complementaria, se pidió a los moradores de las viviendas bajo estudio que capturaran todos los triatominos que detectaran dentro de sus viviendas.
Los ejemplares recolectados fueron llevados al laboratorio para realizar su ubicación taxonómica mediante las claves de Lent y Wygodzinsky (25), así como para realizar su análisis en busca de $T$. cruzi.

\section{Resultados}

Durante el año de estudio, se recolectaron exclusivamente ejemplares adultos de triatominos $(n=457)$, de los cuales, un porcentaje significativamente $(p<0,05)$ mayor $(93 \%)$ eran $M$. longipennis (cuadro 1) y el resto (7\%) T. barberi (cuadro 1). Casi un tercio $(31,29 \%, n=425)$ de $M$. longipennis se recolectaron dentro de los gallineros, el resto $(68,71 \%)$ fue capturado dentro de las viviendas. La cantidad de viviendas con presencia de esta especie varió a lo largo del año de estudio, incrementándose en los meses de abril a junio, en contraste con el número de gallineros con ejemplares, el cual fue similar en la mayoría de los meses (cuadro 2). Por otra parte, tanto el número de ejemplares recolectados en las viviendas como el recolectado en los gallineros se mantuvieron con ligeras variaciones desde febrero hasta septiembre (cuadro 1).

Respecto a $T$. barberi, no se recolectó ningún ejemplar en los gallineros; la totalidad (100\%, $\mathrm{n}=32$ ) de los ejemplares se recolectó dentro de las viviendas, siempre en presencia de, al menos, un ejemplar de $M$. longipennis. A diferencia de lo ocurrido con $M$. longipennis, la cantidad de viviendas con presencia de $T$. barberi fue similar a largo del año de estudio (cuadro 2). Lo opuesto

Cuadro 1. Distribución de los ejemplares de triatominos recolectados a lo largo de un año, en el área de estudio.

\begin{tabular}{|c|c|c|c|c|c|c|c|c|c|c|c|c|}
\hline & \multicolumn{12}{|c|}{ Meses } \\
\hline & Enero & Febrero & Marzo & Abril & Mayo & Junio & Julio & Agosto & Septiembre & Octubre & Noviembre & Diciembre \\
\hline \multicolumn{13}{|c|}{ Meccus longipennis } \\
\hline Viviendas & 0 & 31 & 35 & 38 & 36 & 37 & 34 & 32 & 33 & 16 & 0 & 0 \\
\hline Gallineros & 0 & 15 & 15 & 17 & 20 & 20 & 15 & 14 & 13 & 4 & 0 & 0 \\
\hline \multicolumn{13}{|c|}{ Triatoma barberi } \\
\hline Viviendas & 0 & 0 & 2 & 4 & 9 & 10 & 3 & 2 & 1 & 1 & 0 & 0 \\
\hline Gallineros & 0 & 0 & 0 & 0 & 0 & 0 & 0 & 0 & 0 & 0 & 0 & 0 \\
\hline
\end{tabular}

Cuadro 2. Hábitats con presencia de triatominos, recolectados en el área de estudio.

\begin{tabular}{|c|c|c|c|c|c|c|c|c|c|c|c|c|}
\hline & \multicolumn{12}{|c|}{ Meses } \\
\hline & Enero & Febrero & Marzo & Abril & Mayo & Junio & Julio & Agosto & Septiembre & Octubre & Noviembre & Diciembre \\
\hline \multicolumn{13}{|c|}{ Meccus longipennis } \\
\hline Viviendas & 0 & 10 & 15 & 24 & 36 & 29 & 14 & 16 & 15 & 5 & 0 & 0 \\
\hline Gallineros & 0 & 2 & 2 & 4 & 4 & 3 & 2 & 2 & 2 & 1 & 0 & 0 \\
\hline \multicolumn{13}{|c|}{ Triatoma barberi } \\
\hline Viviendas & 0 & 0 & 1 & 2 & 4 & 3 & 1 & 1 & 1 & 1 & 0 & 0 \\
\hline Gallineros & 0 & 0 & 0 & 0 & 0 & 0 & 0 & 0 & 0 & 0 & 0 & 0 \\
\hline
\end{tabular}


ocurrió en el caso del número de ejemplares recolectado, el cual mostró un incremento en mayo y junio (cuadro 1).

Un poco más de una cuarta parte $(25,65 \%, \mathrm{n}=425)$ de $M$. longipennis y más de la mitad $(53,13 \%, n=32)$ de los ejemplares de $T$. barberi resultaron positivos para la presencia de $T$. cruzi.

\section{Discusión}

Se recolectaron más ejemplares de $M$. longipennis que de $T$. barberi (poco más de $5 \%$ ), de forma significativa $(p<0,05)$. Estos resultados corroboran lo publicado en diversos estudios realizados en el occidente de México sobre la distribución y abundancia de las diversas especies de triatominos ubicadas en el área, en los cuales la especie más abundante y distribuida fue $M$. longipennis $(5-7,9,10)$.

El hecho de que significativamente $(p<0,05)$ más de los ejemplares de $M$. longipennis haya sido capturado dentro de las viviendas, es indicativo de que es una especie que, aun siendo esencialmente peridoméstica y en proceso de adaptación al domicilio humano $(6,7,26)$, comúnmente se mueve de su hábitat peridoméstico (bardas de piedra, gallineros, etc.) hacia las viviendas $(7,8,14)$, lo que incrementa el riesgo de transmisión de $T$. cruzi a los pobladores humanos.

Por otra parte, $T$. barberi fue recolectado exclusivamente en el intradomicilio, como ha sido reportado previamente en otras áreas de México $(27,28)$, lo que podría ser indicativo de riesgo para los habitantes del área. Aparentemente, las poblaciones peridomésticas de esta especie, recolectadas previamente en la misma área geográfica, (7) no se sintieron atraídas a invadir los gallineros y, probablemente, invadieron las viviendas atraídas por la luz.

La cantidad de viviendas con presencia de triatominos, consideradas las dos especies en conjunto, fue significativamente $(p<0,05)$ más alta en los meses de primavera en el hemisferio norte, decreciendo al inicio del verano y de la temporada de lluvias. Aparentemente, la dispersión de estas especies está relacionada con la conjunción entre el incremento en las temperaturas ambientales y la falta de lluvias, lo que favorece su movilidad, en búsqueda de alimento y un microambiente favorable para instalarse $(13,14)$.

Se registró presencia de las dos especies de triatominos desde marzo (incluso desde febrero, de M. longipennis) hasta octubre (a mediados del otoño). El número de ejemplares recolectados de $M$. longipennis fue similar $(p>0,05)$ en todos los meses (excepto en octubre) cuando se capturó esta especie, en tanto que el de $T$. barberi recolectados se incrementó ligeramente en mayo y junio (meses calurosos y previos a temporada de lluvias). Estos resultados fueron similares a lo reportado por Gómez-Hernández (13) para estas dos especies en un área geográfica cercana a la de estudio. Aparentemente, $M$. longipennis se ve menos afectado por las condiciones ambientales que $T$. barberi, una especie más delicada, incluso difícil de mantener aún en condiciones de laboratorio (29).

Los porcentajes, tanto de M. longipennis como de $T$. barberi positivos para $T$. cruzi, fueron similares a lo reportado para estas especies en áreas geográficas cercanas a la de estudio, en el estado de Jalisco (ambas especies) y en los vecinos estado de Nayarit y Colima ( $M$. longipennis) $(6,8,14)$. Los datos obtenidos para $M$. longipennis confirman la importancia vectorial de esta especie en la región, establecida previamente (5,7-9).

Por otra parte, si bien en diversos estudios $(7,10,12,14)$ se asigna a $T$. barberi un papel de vector secundario, en una reciente publicación de compilación (5) sobre la situación de la enfermedad de Chagas en el estado de Jalisco, así como el presente trabajo, se establece el posible papel primario de $T$. barberi como vector en diversas áreas del estado de Jalisco.

En contraste con un estudio previo de vigilancia en el área (7) y otro en Centroamérica (uso de gallineros para vigilancia de poblaciones de T. ryckmani, Zeledón y Ponce, 1972) (30), el éxito de los gallineros para detectar la presencia de los triatominos fue parcial, pues sólo aproximadamente un tercio de ellos fue recolectado en aquéllos. Sin embargo, la implementación de esta medida en conjunción con la búsqueda continua de triatominos dentro de la propia vivienda, permitirían a los habitantes del área de estudio mantener una vigilancia constante de los triatominos domésticos.

\section{Agradecimientos}

A las comunidades del área de Teocuitatlán de Corona por su valiosa participación y apoyo durante el trabajo de campo.

\section{Conflicto de intereses}

Los autores manifiestan que los resultados obtenidos en esta investigación no están relacionados con ningún tipo de intereses. 


\section{Financiación}

Este estudio fue financiado por la Universidad de Guadalajara.

\section{Referencias}

1. Ramsey JM, Tello-López A, Pohls JL. Iniciativa para la vigilancia y el control de la enfermedad de Chagas en la República Mexicana. Cuernavaca: INSP; 2003.

2. Cruz-Reyes A, Pickering-López JM. Chagas disease in Mexico: an analysis of geographical distribution during the past 76 years- A review. Mem Inst Oswaldo Cruz. 2006;101:345-54.

3. Trujillo-Contreras F, Lozano-Kasten F, Soto GM, Hernández GR. Prevalencia de infección a Trypanosoma cruzi en donadores de sangre en el estado de Jalisco, México. Rev Soc Bras Med Trop. 1993;26:89-92.

4. Coll-Cárdenas R, Espinoza-Gómez F, MaldonadoRodríguez A, Reyes-López PA, Huerta-Viera M, RojasLarios F. Active transmission of human Chagas disease in Colima, Mexico. Mem Inst Oswaldo Cruz. 2004;99:363-8.

5. Lozano-Kasten F, Magallón-Gastélum E, Soto-Gutiérrez M, Kasten-Monges M, Bosseno MF, Breniere SF. Conocimiento epidemiológico y situación actual de la enfermedad de Chagas en el estado de Jalisco, México. Salud Pública Méx. 2008;50:508-15.

6 Martínez-lbarra JA, Bárcenas-Ortega NM, NoguedaTorres B, Alejandre-Aguilar R, Rodríguez ML, MagallónGastélum E, et al. Role of two Triatoma (Hemiptera: Reduviidae: Triatominae) species in the transmission of Trypanosoma cruzi (Kinetoplastida: Trypanosomatidae) to man in the west coast of Mexico. Mem Inst Oswaldo Cruz. $2001 ; 96: 141-4$.

7. Martínez-Ibarra JA, Grant-Guillén Y, Morales-Corona ZY, Haro-Rodriguez S, Ventura-Rodríguez LV, NoguedaTorres B, et al. Importance of species of Triatominae (Heteroptera, Reduviidae) in the risk of transmission of Trypanosoma cruzi in Western Mexico. J Med Entomol. 2008;45:476-82.

8. Espinoza-Gómez F, Maldonado-Rodríguez A, CollCárdenas R, Hernández-Suárez CM, Fernández-Salas I. Presence of Triatominae (Hemiptera: Reduviidae) and risk of transmission of Chagas disease in Colima, México. Mem Inst Oswaldo Cruz. 2002;97:25-30.

9. Magallón-Gastélum E, Lozano-Kasten F, Bosseno MF, Cárdenas-Contreras R, Ouaissi A, Breniere SF. Colonization of rock pile boundary walls in fields by sylvatic Triatomines (Hemiptera: Reduviidae) in Jalisco state, Mexico. J Med Entomol. 2004.;41:484-8.

10. Magallón-Gastélum E, Lozano-Kasten FJ, SotoGutiérrez M, Flores-Pérez A, Sánchez B, Espinoza B, et al. Epidemiological risk for Trypanosoma cruzi transmisión by species of Phyllosoma complex in the occidental part of Mexico. Acta Trop. 2006;97:331-8.

11. Breniere SF, Pietrokovsky S, Magallón-Gastélum E, Bosseno MF, Soto MM, Ouaissi A, et al. Feeding patterns of Triatoma longipennis Usinger (Hemiptera, Reduviidae) in peridomestic habitats of a rural community in Jalisco state, Mexico. J Med Entomol. 2004;41:1015-20.

12. Walter A, Lozano-Kasten F, Bosseno MF, CastilloRuvalcaba EG, Soto-Gutiérrez M, Montaño-Luna EC, et al. Peridomiciliary habitat and risk factors for Triatoma infestation in a rural community of the Mexican occident. Am J Trop Med Hyg. 2007;76:508-15.

13. Gómez-Hernández C, Rezende-Oliveira K, CortésZárate A, Cortés-Zárate E, Trujillo-Contreras F, Ramírez LE. Prevalence of triatomines (Hemiptera: Reduviidae: Triatominae) infected by Trypanosoma cruzi; seasonality and distribution in the Ciénaga region of the state of Jalisco, Mexico. Rev Soc Bras Med Trop. 2008;41:257-62.

14. Breniere SF, Bosseno MF, Magallón-Gastélum E, Castillo-Ruvalcaba EG, Soto MM, Montaño-Luna EC, et al. Peridomestic colonization of Triatoma longipennis (Hemiptera, Reduviidae) and Triatoma barberi (Hemiptera: Reduviidae) in a rural community with active transmission of Trypanosoma cruzi in Jalisco state, Mexico. Acta Trop. 2007;101:249-57.

15. Martínez-lbarra JA, Grant-Guillén Y, Martínez-Grant DM. Feeding, defecation, and development times of Meccus longipennis Usinger, 1939 (Hemiptera: Reduviidae: Triatominae) under laboratory conditions. Mem Inst Oswaldo Cruz. 2003;98:899-903.

16. Martínez-Ibarra JA, Grant-Guillén Y, Nogueda-Torres B, Trujillo-Contreras F. Influence of the blood meal source on the biology of Meccus longipennis Usinger 1939 (Hemiptera: Reduviidae) under laboratory conditions. J Am Mosq Control Assoc. 2004;20:328-30.

17. Martínez-Ibarra JA, Grant-Guillén Y, Morales-Corona ZY, Martínez-Grant JA, Trujillo-Contreras F. Evaluation of risk factors for rural infestation by Meccus longipennis (Hemiptera: Triatominae), a Mexican vector of Chagas disease. J Am Mosq Control Assoc. 2005;21:421.

18. Wisnivesky-Colli C. Triatominos vectores secundarios de Trypanosoma cruzi: su domiciliación potencial. Talleres (Venezuela). 1994;3:83-9.

19. Schweigmann NJ, Pietrokovsky S, Conti O, Bottazzi V, Canale D, Wisnivesky-Colli C. The interaction between poultry and Triatoma infestans Klug, 1834 (Hemiptera: Reduviidae) in an experimental model. Mem Inst Oswaldo Cruz. 1995;90:429-31.

20. Cécere MC, Gürtler RE, Canale D, Chuit R, Cohen JE. El papel del peridomicilio en la eliminación de Triatoma infestans de comunidades rurales argentinas. Bol Ofic Sanit Panam. 1996;121:1-9.

21. Cécere MC, Gürtler RE, Canale D, Chuit R, Cohen JE. Effects of chickens on the prevalence of infestation and population density of Triatoma infestans in rural houses of north-west Argentina. Med Vet Entomol. 1997;11:383-8.

22. Gürtler RE, Cécere MC, Rubel DN, Schweigmann N. Determinants of the domiciliary density of Triatoma infestans, vector of Chagas disease. Med Vet Entomol.1999;6:75-83.

23. Epi Info. Users manual. Sixth edition. Atlanta: CDC; 2006.

24. Pinchin R, Fanara DM, Castleton CW, Oliveira-Filho AM. Comparison of techniques for detection of domestic infestations with Triatoma infestans in Brazil. Trans R Soc Trop Med Hyg. 1981;75:691-4.

25. Lent H, Wygodzinsky P. Revision of the Triatominae (Hemiptera:Reduviidae) and their significance as vectors of Chagas' disease. Bull Am Mus Nat Hist. 1979;163:123520. 
26. Zeledón R. Vectores de la enfermedad de Chagas y sus características ecofisiológicas. Interciencia. 1983;8:384-95.

27. Ramsey JM, Ordóñez R, Cruz-Celis A, Alvear AL, Chávez V, López $\mathbf{R}$, et al. Distribution of domestic Triatominae and stratification of Chagas disease transmission in Oaxaca, México. Med Vet Entomol. 2000;14:19-30.

28. Salazar-Schettino PM, De Haro-Arteaga I, CabreraBravo M. Tres especies de triatominos y su importancia como vectores de Trypanosoma cruzi en México. Medicina (Buenos Aires). 2005;65:63-9.
29. Martínez-lbarra JA, Nogueda-Torres B, Paredes E, Alejandre-Aguilar R, Solorio-Cibrián M, Barreto SP, et al. Development of Triatoma rubida sonoriana, Triatoma barberi, and Meccus mazzottii (Heteroptera, Reduviidae) under laboratory conditions. J Am Mosq Control Assoc. 2005;21:310-5.

30. Monroy C, Marroquín R, Rodas A, Rosales R, Jaenson TG. Dispersion and colonization of Triatoma ryckmani (Hemiptera: Reduviidae) in artificial environments in a semiarid region of Chagas disease endemic area in Guatemala. Acta Trop. 2004;91:45-51. 